\title{
Torniquetes: ¿cómo clasificarlos? Propuesta de clasificación de acuerdo con su mecanismo de acción y sitio de aplicación
}

\author{
Omar Yassef Antúnez-Montes ${ }^{1,2 *}$, Carlos Antonio Contreras-Cordero ${ }^{1}$ y Carlos José Ascencio-Guerrero ${ }^{2}$ \\ ${ }^{1}$ Departamento de capacitación, Concord Defense México; ${ }^{2}$ Escuela Superior de Medicina, Instituto Politécnico Nacional. Ciudad de México, México
}

\begin{abstract}
Resumen
Introducción: Los torniquetes (TQ) son dispositivos cuyo fin es detener el sangrado. Existe una gran cantidad de TQ que ofrecen isquemia mediante mecanismos semejantes, sin embargo no hemos encontrado una clasificación de ellos que se base en su mecanismo de acción para lograrlo. Objetivos: Identificar los diferentes mecanismos de acción de los TQ y clasificarlos de acuerdo con este, identificar los diferentes sitios de aplicación de los TQ y clasificarlos con base en este. Material y métodos: Se llevó a cabo una investigación bibliográfica sobre el uso, diseño, efectividad y actualidad de los $T Q$ en el mercado mediante bases de datos y descriptores. Criterios de inclusión: artículos publicados desde el año 2000 hasta el 2018 en inglés y español. Criterios de exclusión: artículos publicados en revistas no indexadas. Resultados: Según su sitio de aplicación se agruparon como TQ de extremidades y TQ para hemorragias de la unión. Identificamos el mecanismo de ajuste primario como un sistema de palanca para colocarlo sobre la extremidad. Según su mecanismo de acción se propone clasificarlos como 1) molinete, 2) neumáticos, 3) rolled-up, 4) safe-clip y 5) tornillo de compresión. Conclusiones: Los $T Q$ han tenido cambios importantes en el diseño del ajuste primario. Su mecanismo de acción no ha tenido cambios sustanciales, es así que reconocemos cinco grupos para TQ en el mercado: neumáticos, molinete, safe-clip, rolled-up y tornillo de compresión. Los más seguros y eficientes son los neumáticos y de molinete. Clasificarlos ayudará a determinar cuál es mejor según el contexto en que se encuentre el personal. Entender el mecanismo de acción facilitará su reconocimiento, fabricación, investigación e innovación tecnológica.
\end{abstract}

Palabras clave: Trauma de extremidades. Hemorragia de la unión. Torniquetes. Torniquete de combate. Clasificación de torniquetes. Control del sangrado.

\section{Tourniquets: How to classify them? Classification proposal according to its mechanism of action and application site}

\begin{abstract}
Introduction: Tourniquets (TQ) are devices whose purpose is to stop bleeding. There is a large number of TQ that offer ischemia through similar mechanisms, however we have not found a classification of them based on their mechanism of action to achieve it. Objectives: Identify the different mechanisms of action of the TQ and classify them according to their mechanism of action, identify the different application sites of the $T Q$ and classify them according to their application site.
\end{abstract}

\section{Correspondencia:}

*Omar Yassef Antúnez-Montes

E-mail: antunezyassef@gmail.com
Fecha de recepción: 20-03-2019

Fecha de aceptación: 18-09-2019 DOI: 10.24875/REIE.19000023
Disponible en internet: 06-11-2019 Rev Educ Investig Emer. 2019;1(3):89-97 www.medicinadeemergencias.com

2604-6520 @ 2019 Sociedad Mexicana de Medicina de Emergencias, AC. Publicado por Permanyer México SA de CV. Este es un artículo open access bajo la licencia CC BY-NC-ND (http://creativecommons.org/licenses/by-nc-nd/4.0/). 
Materials and methods: A bibliographic research was carried out on the use, design, effectiveness and topically of TQ in the market through databases and descriptors. Inclusion criteria: articles published from 2000 to 2018 in English and Spanish. Exclusion criteria: articles published in non-indexed journals. Results: According to their application site they were grouped as TQ of extremities and TQ for hemorrhages of the union. We identify the mechanism of primary adjustment as a lever system to place it on the limb. According to its mechanism of action it is proposed to classify them as (1) windlass, (2) pneumatic, (3) rolled-up, (4) safe-clip, and (5) compression screw. Conclusions: The TQ have had important changes in the design of the primary adjustment. Its mechanism of action has not had substantial changes, so we recognize five groups for TQ in the market: pneumatic, windlass, safe-clip, rolled-up and compression screw. The safest and most efficient are pneumatic and windlass TQ. Classifying them will help determine which is best according to the context in which the personnel is located. Understanding the mechanism of action will facilitate its recognition, manufacturing, research and technological innovation.

Key words: Limb trauma. Joint hemorrhage. Tourniquets. Combat tourniquet. Tourniquet classification. Bleeding control.

\section{Historia}

El uso del torniquete (TQ) ha sido motivado por las guerras y posteriormente introducido al ambiente quirúrgico. Se tiene registro del uso de TQ para el control de hemorragias en el muslo por el imperio romano (199 AEC-500 EC) hechos de bronce que probablemente se encontraban recubiertos por cuero para mayor comodidad del paciente ${ }^{1}$. A Ambrose Paré (1510-1590) se le atribuye la primera recomendación sobre su uso quirúrgico, sin embargo el término torniquete se disputa entre Paré y Jean Louis Petit en 1718 derivado del francés tourner (que gira) usando estos dispositivos para las amputaciones de miembros inferiores ${ }^{2}$. Paré colocó un tornillo con almohadilla sobre el vaso principal de una extremidad apretándolo con el fin de incrementar la tensión sobre una correa circunferencial, en el siglo XVII William Fabry y Étienne Morel utilizaron un molinete de giro con el objetivo de apretar aún más y de forma uniforme la banda circunferencial de constricción. Lister y Esmarch utilizaron TQ a mediados del siglo XIX para introducir la cirugía con campos limpios. En 1904 Cushing abandona los molinetes originales para introducir un sistema neumático que se podía aplicar y retirar con mayor facilidad, y permite controlar la presión de forma más uniforme en comparación con los modelos anteriores $^{3}$. Todos los TQ desde la antigüedad hasta el día de hoy mantienen características en común y sus modificaciones no han sido sustanciales a lo largo de la historia, pues uno de los principios para incrementar la presión, «el molinete», perdura hasta el día de hoy. El desarrollo de los TQ ha sido motivo de estudio en los últimos 18 años gracias a los conflictos armados, donde las heridas exanguinantes son causa elevada de mortalidad. Se tiene registrado por los US Army Rangers una mayor incidencia de heridas de bala en la guerra civil somalí (55\%) el 3 y 4 de octubre de 1993 que en la guerra de Vietnam (30\%), 19551975. En Somalia el $14 \%$ de las heridas fueron en pelvis, en Vietnam fueron el $2 \%$. Para las heridas en extremidades los porcentajes son iguales: Somalia $7 \%$, Vietnam $7 \% 4,5$. Durante la operación «Libertad» iraquí y la operación «Libertad duradera» en África en el periodo de octubre de 2001 a junio de 2011 se registró una mortalidad asociada en gran medida a la hemorragia en un $90.9 \%$ de las bajas. Los lugares anatómicos de hemorragia letal fueron en un $67.3 \%$ el tronco (36\% torácica y $64 \%$ abdominopélvica), seguido de hemorragia de la unión con un 19.2\% (cervical $39.2 \%$, axilar e inguinal $60.8 \%$ ) y de las extremidades periféricas con un $13.5 \%{ }^{6}$. Los TQ modernos más representativos como hoy los conocemos fueron estudiados por el United States Army Institute of Surgical Research a finales de 2005, demostrando que los más eficientes para el combate eran los modelos CAT, SOFTT y EMT, que disminuían la mortalidad en un $85 \% 6,7$. Al analizar el funcionamiento de los TQ nos dimos cuenta de que a pesar de existir una gran cantidad de modelos, todos guardaban semejanzas en el sistema mecánico para detener el sangrado, pero hasta el momento no hemos encontrado en la literatura una clasificación de los TQ por mecanismo de acción para detener el sangrado. Es por esta razón que nos dimos a la tarea de analizar los sistemas mecánicos y definir similitudes entre ellos para poder clasificarlos.

\section{Objetivos}

Identificar los diferentes mecanismos de acción de los TQ y clasificarlos de acuerdo a él, identificar los diferentes sitios de aplicación de los TQ y clasificarlos según este. 


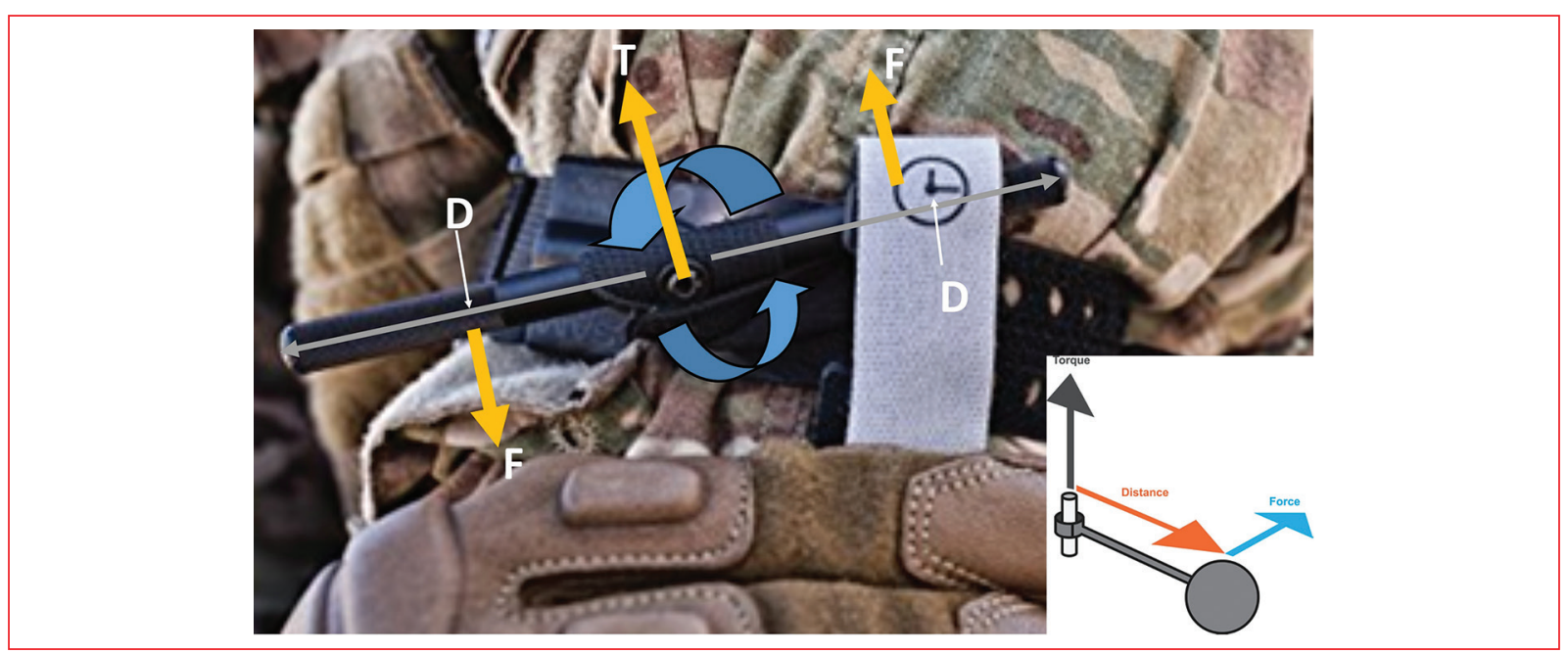

Figura 1. Al girar el molinete provocamos fuerza de torque que se va a traducir en una ventaja mecánica incrementando de manera proporcional y uniforme la fuerza en la correa circunferencial previamente colocada por el sistema de ajuste primario. Torniquete SAM con un mecanismo de ajuste primario de hebilla y mecanismo de acción de molinete. D: distancia; T: torque; F: fuerza.

\section{Material y métodos}

Para proponer la siguiente clasificación llevamos a cabo una investigación bibliográfica sobre el uso, diseño, efectividad y actualidad sobre los $T Q$ en el mercado mediante las bases de datos, Medline, PubMed, LILACS, Cochrane, Google Académico, NHS, Centre for Reviews and Dissemination y Embase-National Research Register, mediante los siguientes descriptores: trauma de extremidades, trauma vascular, torniquete, control de hemorragia, combate, hemostasia, desastres, servicios de urgencia/emergencia. Como criterio de inclusión: artículos publicados desde el año 2000 hasta el 2018 (excepto para las referencias históricas) en inglés y español, artículos enfocados al diseño, clasificación y comparación de los TQ. Criterios de exclusión: artículos publicados en revistas no indexadas. Una vez reunidos los datos se analizaron los modelos de TQ citados a continuación como los más comunes en el mercado buscando similitudes en su funcionamiento para detener el sagrado, así como su sitio de aplicación.

\section{Mecanismo de acción de los torniquetes}

Definimos el mecanismo de acción de un TQ como el componente o componentes mecánicos que acciona el operador en el dispositivo con el objetivo de incrementar la presión administrada en el ajuste primario. Los TQ incrementan esta presión gracias a los principios de las máquinas simples en conjunto con sistemas mecánicos agregados. El fundamento anterior es lo que nos permite agruparlos y clasificarlos como proponemos debido a que el éxito del dispositivo para detener el sangrado se basa en el mecanismo de acción que genera incremento uniforme de la presión radial al accionarlo, imposible lograrlo apretando únicamente una correa con las manos sobre la extremidad afectada si no cuenta con el principio mecánico correcto, como se demostró al comparar los TQ improvisados sin molinete, que fallaron para detener el sangrado en un 99 y $100 \%$ en detener el pulso distal ${ }^{8}$. Se describen los mecanismos de acción propuestos para clasificar los dispositivos.

\section{Molinete}

Como ya se mencionó, el molinete ha formado parte importante del control de hemorragias en dispositivos improvisados como comerciales, los principios físicos del molinete se basan en la torsión (torque) que ofrece una ventaja mecánica (relación entre la fuerza aplicada y la fuerza resultante) de modo que la dirección y magnitud de la fuerza ejercida en la barra genera una fuerza de torque transferida de manera directamente proporcional a toda la correa circunferencial, incrementando uniformemente la presión radial para el colapso de los vasos sanguíneos. Es así que la potencia $(P)$ la ejercemos mediante el torque en el molinete, el fulcro (F) se encuentra en la correa que une al molinete por el centro funcionando como apoyo con cada rotación y finalmente la resistencia (R) la encontramos en los tejidos rodeados de manera circunferencial ${ }^{9}$ (Fig. 1). 


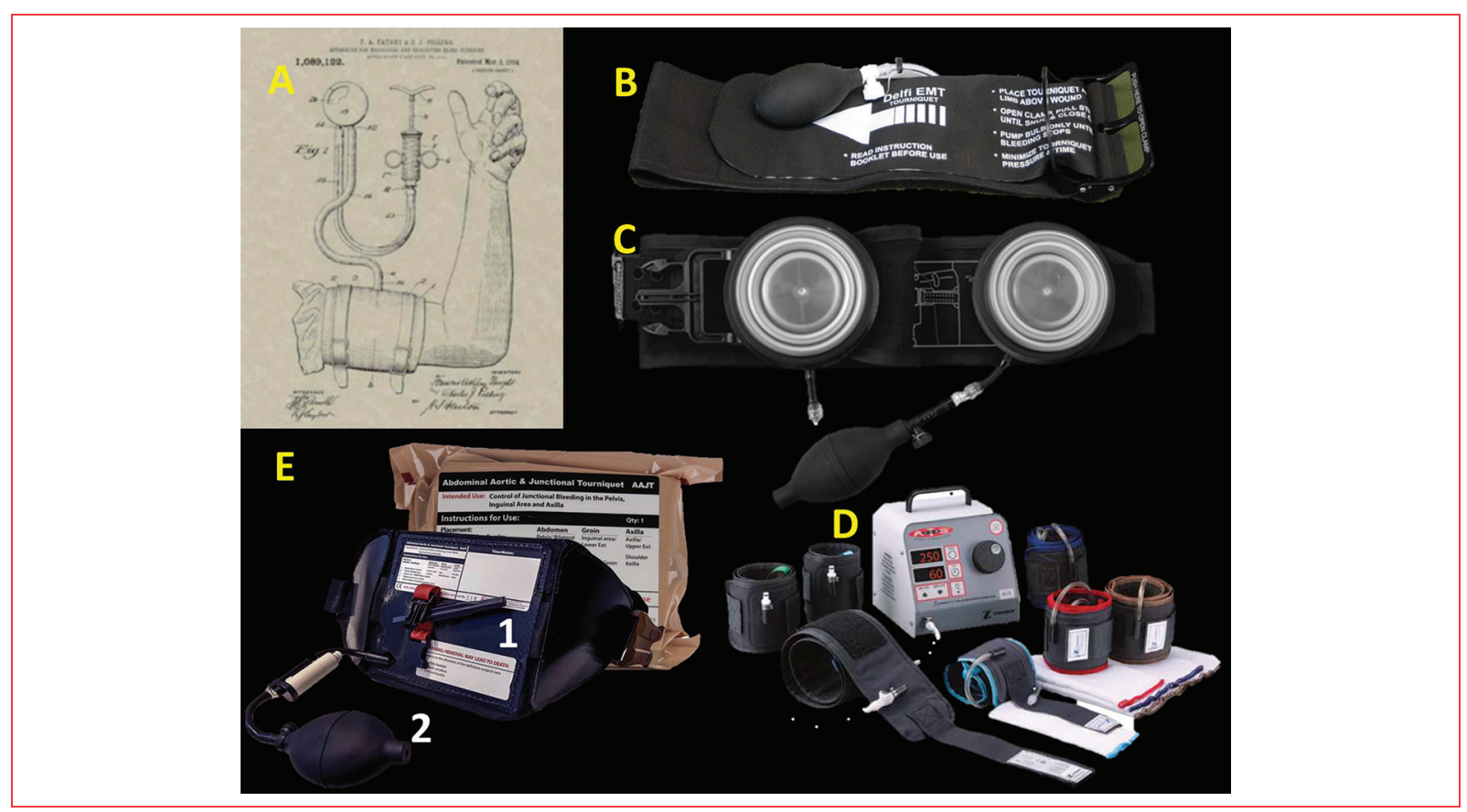

Figura 2. A: Torniquete (TQ) neumático de 1914, a partir del diseño de Cushing. La presión se monitorizaba por medio de un manómetro conectado al circuito. Diferentes T0 neumáticos en el mercado, para extremidades. B: EMT Delfi Medical, hemorragias de la unión. C: SAM JT, hemorragias de la unión. E: AAJT, este torniquete es mixto 1: molinete y 2: neumático. D: los automáticos pueden variar en marcas y características, su uso es quirúrgico. Todos cuentan con perillas para su funcionamiento manual, estos dispositivos tienen el mecanismo de acción más seguro y eficiente.

\section{Neumáticos}

En 1904 Cushing introduce el sistema neumático en los TQ permitiéndole controlar la presión del dispositivo manualmente ${ }^{10}$, los TQ neumáticos son muy usados en procedimientos quirúrgicos ortopédicos y se ha destacado su participación en la guerra desde $2005^{7}$. Su mecanismo de acción está fundamentado en un manguito o vejiga inflable dentro de una funda textil (nailon) que rodea las extremidades como un brazalete, al inflar el manguito la presión se distribuye uniformemente en tejidos subyacentes hacia los vasos sanguíneos venciendo la presión sistólica colapsándolos; el ancho del manguito es superior a los TQ para extremidades no neumáticos, la forma de inflarse con aire puede lograrse vía manual o automática teniendo además la oportunidad de visualizar y controlar los $\mathrm{mmHg}$ suministrados al manguito, manteniendo un control individual de la presión administrada a cada paciente ${ }^{11,12}$ (Fig. 2).

\section{Sistema de palanca}

Este mecanismo de acción se desempeña mediante «estirar/traccionar, envolver y abrochar/asegurar» el
TQ independientemente del material, características físicas y dimensiones de los productos, que entre ellos es ya bastante variada; la mayoría son elásticos. El incremento de presión se logra al aumentar la tensión conforme se va enrollando, estirando y apretando el $T Q$ con cada vuelta que se le da envolviendo la extremidad en cuestión, el mecanismo de acción es igualmente una aplicación de la palanca que ya vimos en el sistema de ajuste primario al colocar los TQ. En un extremo aplicamos la $\mathrm{P}$ para tensar el dispositivo, el $\mathrm{F}$ se encuentra en una hebilla, gancho, o la misma extremidad que nos va a servir como apoyo para incrementar y mantener la tensión que se da con cada vuelta, una fuerza de $\mathrm{R}^{9}$ en los tejidos que tendremos que vencer para detener el sangrado; finalmente su extremo distal tiene que ser asegurado para evitar que se afloje el TQ y pierda la tensión administrada (Fig. 3).

\section{Trinquete}

El trinquete es un mecanismo rotatorio compuesto por una rueda dentada, un gatillo de freno y un soporte donde se mantiene el mecanismo, la rueda dentada se 


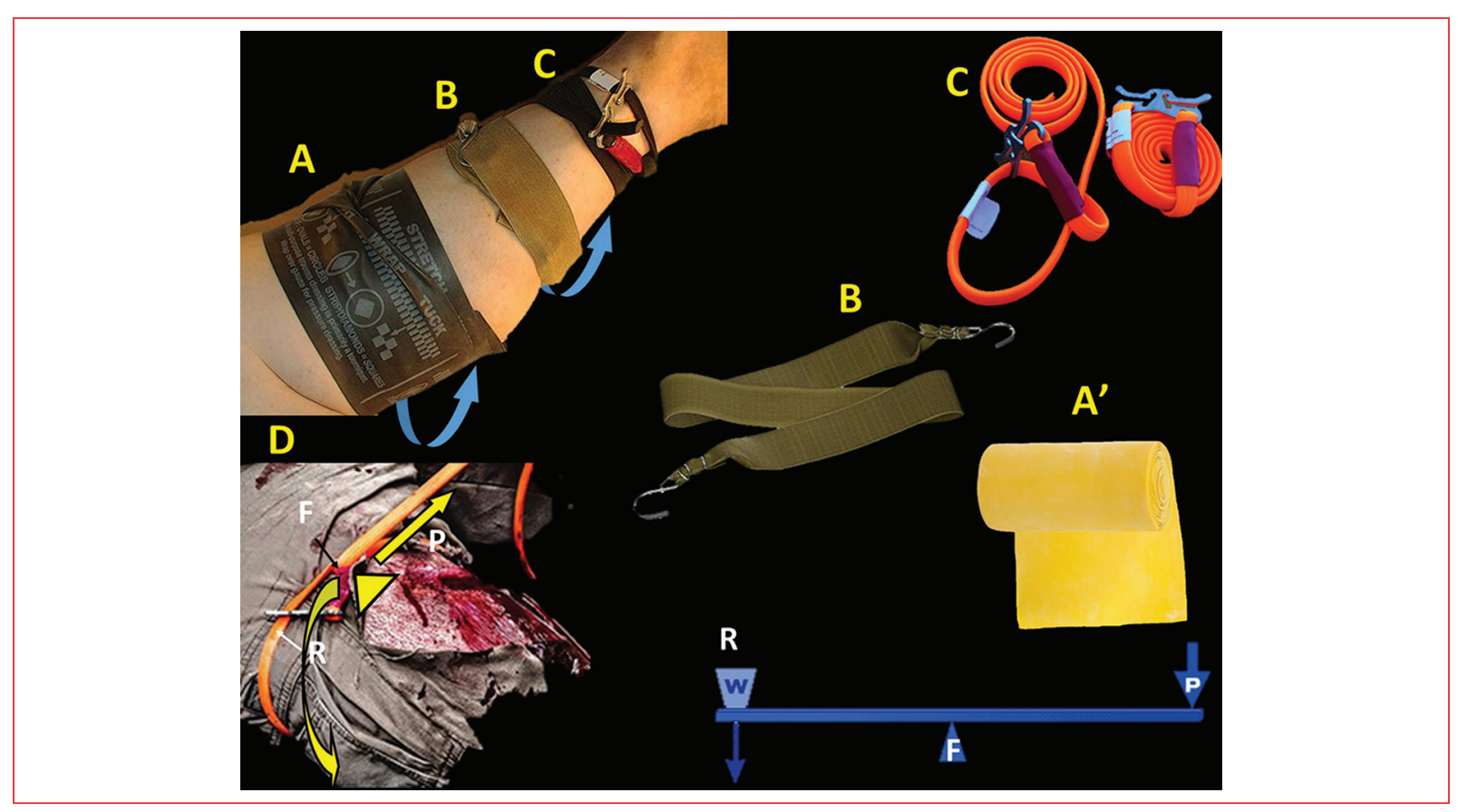

Figura 3. Torniquetes rolled-up. Su mecanismo de acción se basa en el sistema de palanca y la fuerza aplicada mientras se tracciona/estira, se envuelve y se fija al finalizar su colocación. Tienen la desventaja de que al estirarse se reduce su superficie, lo que hace que la fuerza aplicada sea inversamente proporcional al ancho del dispositivo. A: SWAT. A': venda de Smarch, muy similar al SWAT. B: TK4-L. C: RATS. D: aplicación del RATS, se puede apreciar que durante la aplicación de todos estos dispositivos se necesita de los elementos de la palanca de primer grado. P: potencia; R: resistencia; F: fulcro.

acciona por medio de una palanca o un mecanismo similar al molinete que permite el giro de la rueda dentada hacia una dirección pero lo impide al contrario gracias al gatillo de freno. En el caso de los TQ la rotación de la rueda dentada en sentido horario incrementa la tensión circunferencial de la correa sobre el tejido, así que cada vez que se acciona el mecanismo de trinquete la tensión circunferencial incrementa en la extremidad afectada proporcionando una ventaja mecánica (Fig. 4).

\section{Tornillo}

Mecanismo de acción para detener el sangrado basado en una máquina simple conocida como tornillo, el cual recibe un movimiento giratorio (aplicado a un volante o barra horizontal) que es transformado en un movimiento rectilíneo, mediante un mecanismo de tornillo y tuerca. La F aplicada por la longitud de la circunferencia del volante ha de ser igual a la fuerza resultante por el avance del tornillo, el avance de la porción inferior del tornillo tiene como objeto vencer la
R que presenta un sitio anatómico específico para detener el sangrado en una región anatómica (Fig. 5).

\section{Resultados \\ Protocolo de búsqueda}

La búsqueda se realizó en Medline, PubMed, LILACS, Cochrane, Google Académico, NHS, Centre for Reviews and Dissemination y Embase-National Research Register, con un total de 150 artículos arrojados en la búsqueda y una selección final de 47 artículos, se excluyeron 103 artículos por no brindar datos relevantes sobre su clasificación, diseño, desempeño comparativo, errores de fabricación o información repetitiva sobre el uso del dispositivo.

\section{Clasificando los torniquetes por sitio de aplicación y mecanismo de acción}

Se agrupan en dos grandes universos según el sitio de aplicación del dispositivo como: TQ de extremidades y TQ para hemorragias de la unión. 


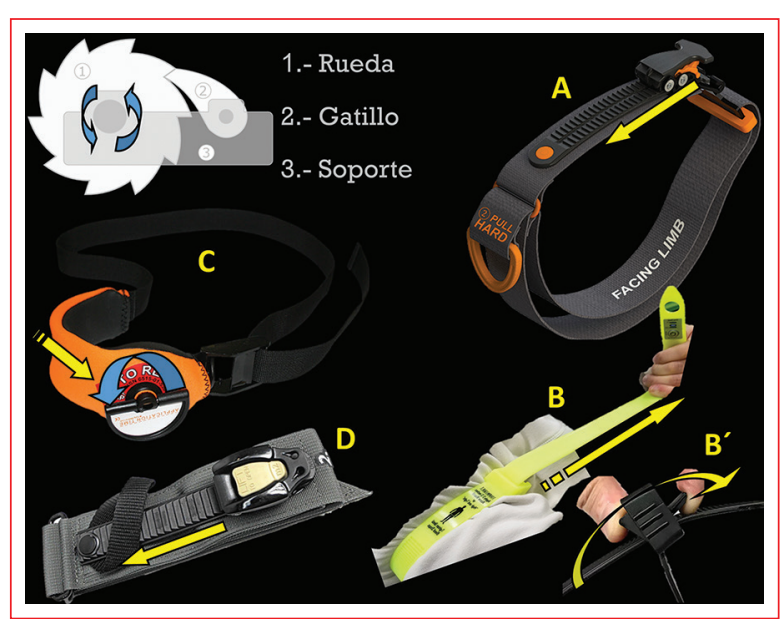

Figura 4. Torniquetes que incluyen el mecanismo de acción del trinquete. Los sistemas de ajuste primario permiten al trinquete ajustarse por medio de palancas como en THOR (A) y RMT (D). C: cabeza de rotación turn key para el modelo MAT. B: STAT la fuerza necesaria para detener el sangrado dependerá de la aplicada por el usuario, en este caso no existe ninguna ventaja mecánica que haga directamente proporcional la fuerza aplicada únicamente el sistema de gatillo (B') que impide la regresión del sistema.

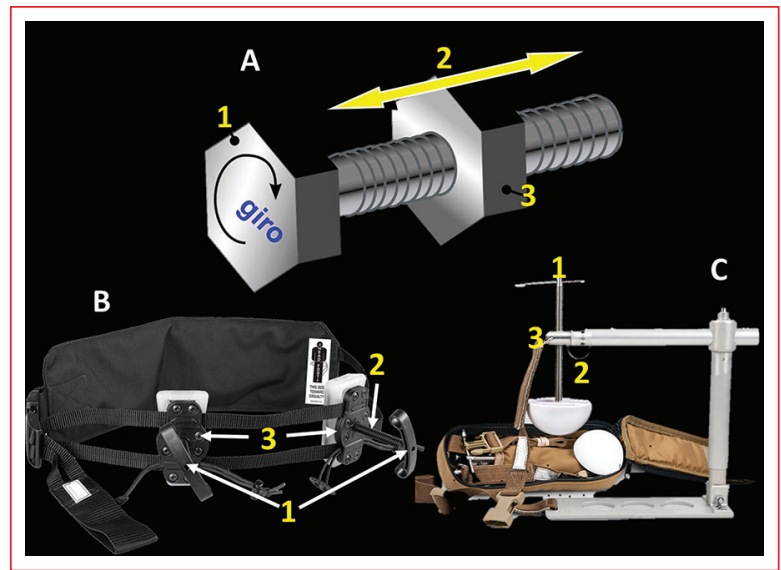

Figura 5. Torniquete para hemorragias de la unión y su mecanismo de acción por medio de la máquina simple del tornillo. A: tornillo: el giro (1) de la cabeza origina el avance (2) rectilíneo del dispositivo a través de una tuerca (3) para vencer la resistencia sobre los tejidos. Modelos JETT (B) y CROC (C) (se muestra por motivos de enseñanza).

Los TQ de extremidades son dispositivos médicos que aplican presión radial en la circunferencia de extremidades superiores o inferiores para colapsar los vasos sanguíneos y ocluir temporalmente la irrigación y drenaje del extremo distal al TQ ${ }^{13}$.
Los TQ para hemorragias de la unión son aquellos dispositivos que tienen como objetivo controlar el sangrado en las áreas de unión al tronco y sus apéndices en caso de presentarse hemorragia en la región de unión cervical, pélvica, axilar e inguinal como sitios de difícil control para heridas exanguinantes y donde no es posible aplicar un TQ de extremidades ${ }^{14}$.

\section{Mecanismo de acción}

Determinamos que durante la aplicación del TQ existen dos momentos importantes para su correcta colocación, no descritos anteriormente en la literatura como factores cruciales en el funcionamiento del dispositivo, los cuales denominamos «sistema de ajuste primario» y «mecanismo de acción».

Podemos reconocer cinco tipos de mecanismos de acción compartidos por los TQ en el mercado bajo los cuales agrupamos los dispositivos, hemos asignado nombres a cada uno de los grupos por el principio que comparten y algunos ejemplos de TQ para cada grupo (Tabla 1).

1. Molinete/varilla: CAT, SOFT y SAM TQ.

2. Sistema de palanca, rolled-up (enrollados): SWAT-T,

RATS, TK4-L, SMARCH y Hemodyne.

3. Trinquete, safe-clip: STAT, THOR, RMT y MAT.

4. Neumáticos: EMT, ATS, SAMJT y AAJT (mixto).

5. Tornillo de compresión: JETT y CROC.

\section{Sistema de ajuste primario}

Todos los TQ se colocan mediante un ajuste primario que se logra por la tracción de correas adheribles, cuerdas, bandas elásticas, etc. Su único objetivo es mantener el dispositivo fijo a la extremidad, pelvis o tórax, este ajuste primario lo vamos a lograr por el principio de palanca. Cuando traccionamos el extremo distal del $T Q$ para ajustarlo en la extremidad generamos una $P$ que recorre apoyada sobre una hebilla funcionando como $\mathrm{F}$ por donde pasamos un extremo del dispositivo a fin de vencer la $\mathrm{R}$ de los tejidos (Fig. 6).

\section{Discusión}

Anteriormente se ha denominado "sistema de ajuste» a los mecanismos que se accionan para incrementar la presión en extremidades mediante trinquete (RMT), molinete (CAT, SOFT) y dispositivos elásticos (SWAT) ${ }^{15}$, definición que consideramos es más adecuada para la colocación de los dispositivos sobre su sitio de acción mas no con el objeto de detener el sangrado, que es lo 
Tabla 1. Clasificatión de los torniquetes según su mecanismo de acción

\begin{tabular}{|l|l|l|l|l|}
\hline & \multicolumn{3}{|c|}{ Mecanismo de acción } \\
\hline Sitio de aplicación & (A) Molinete & (B) Neumáticos & Rolled-Up & Safe-Clip \\
\hline TO de extremidades & CAT & (Manuales) EMT & RATS & STAT \\
\hline & SOFT & (Automáticos) ATS & TK4-L & THOR \\
\hline & SAM & & SWAT-T & RMT \\
\hline TQ hemodyne & Smarch & \\
\hline & & & & \\
\hline & Tornillo de compresión & & & \\
\hline & JETT & SAM JT & \\
\hline
\end{tabular}

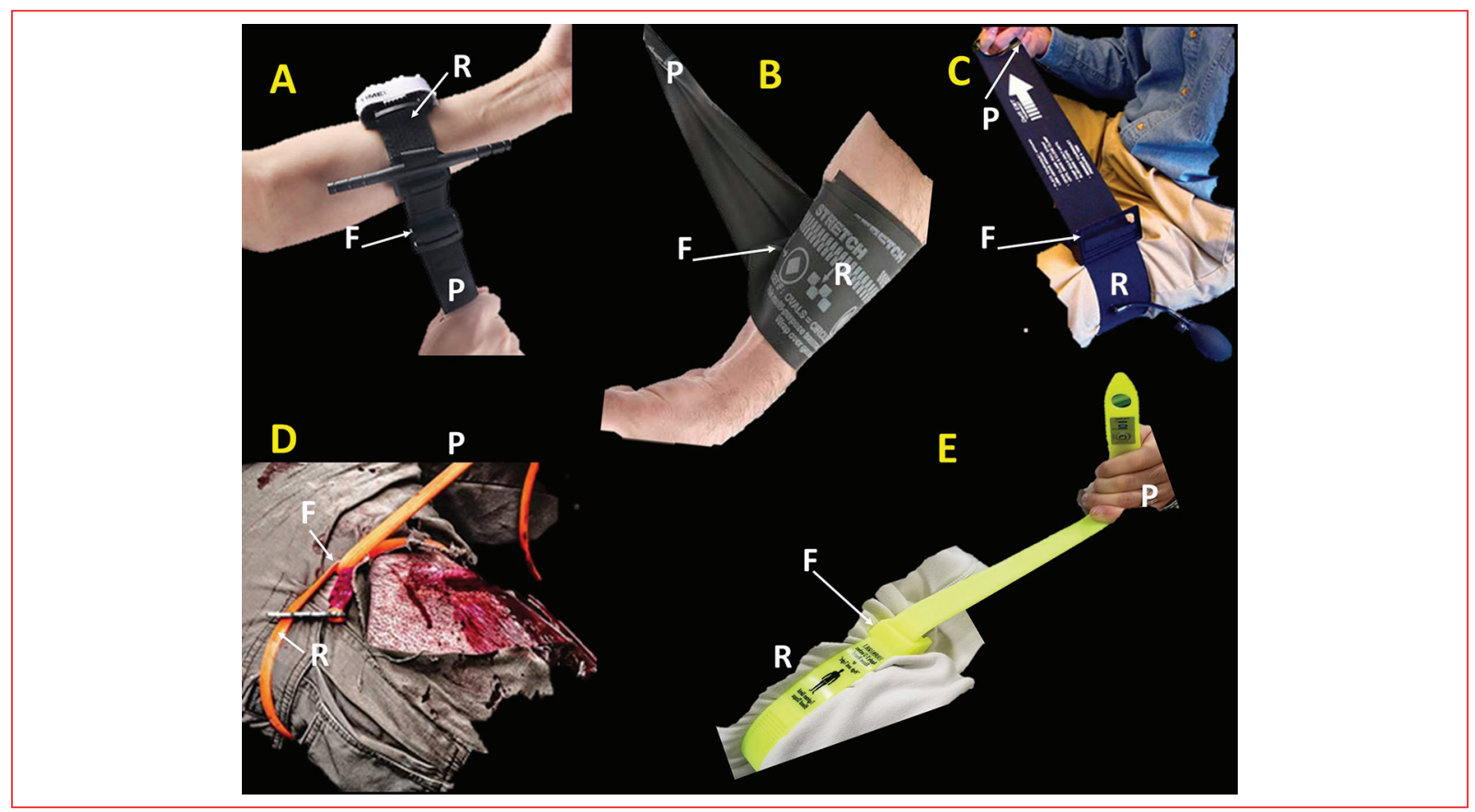

Figura 6. Sistema de ajuste primario. Todos los torniquetes en el mercado se colocan y ajustan mediante mecanismos de palanca, este ajuste no detiene el sangrado en su totalidad, sino que necesita de un mecanismo de acción que le proporcione ventaja mecánica. A: CAT. B: SWAT. C: EMT. D: RATS TQ. E: STAT. P: potencia; R: resistencia; F: fulcro.

que exponemos en este artículo como «mecanismo de acción» de los TQ. Para nosotros es importante reconocer el «sistema de ajuste primario» como un momento crucial en la colocación de un TQ porque es donde se han dado cambios significativos en el diseño de los dispositivos. Reconocemos la palanca de primer grado como el principio físico que ofrece ventaja mecánica ${ }^{9}$ al momento de colocar un torniquete, las mejoras en los diseños deberían incluir un sistema de ajuste primario sencillo y rápido junto a mecanismos de acción más eficientes. El mecanismo de acción de los TQ se ha mantenido sin cambios importantes a lo largo de la historia. Todos los TQ en el mercado diseñados hasta el día de hoy pueden agruparse en los cinco rubros que proponemos. Los TQ de molinete son los más usados en el trabajo de campo por su durabilidad, ajuste primario 
sencillo, mecanismo de acción eficiente, además de necesitar poco entrenamiento para su uso ${ }^{11,16}$; sin embargo, se ha identificado que el molinete tiende a aplicar presiones más altas para detener el sangrado debido a que el molinete tiene que completar vueltas de 180 grados hasta asegurar la varilla, si a mitad de una vuelta se detiene el sangrado tenemos que completar el giro aplicando mayores presiones incrementando la probabilidad de daño, por el contrario retroceder la varilla media vuelta para asegurar el molinete nos dará menores presiones, insuficientes para controlar el sangrado ${ }^{15,17-19}$.

En múltiples estudios se ha demostrado que los mecanismos de acción que clasificamos son eficientes para detener el flujo sanguíneo cuando son aplicados de manera correcta y en un ambiente controlado, hay que mencionar que a pesar de lograr detener el sangrado sus porcentajes de éxito pueden ser variables. Como ejemplos: el mecanismo de acción rolled-up, donde el TQ SWAT falló el $70 \%$ de las veces para detener el sangrado y es difícil asegurar la porción distal para que no se afloje; el modelo TK4-L presentó fallas mecánicas catastróficas, rompiéndose durante su aplicación; los mecanismos de trinquete fallan al tener contacto con arena y dependen del buen funcionamiento de sus componentes ${ }^{15,18,19}$. En contraste el molinete ha demostrado ser efectivo en el $100 \%$ de los modelos con diferencias mínimas entre ellos ${ }^{18-21}$. Al estudiar comparativamente la efectividad del molinete frente a los neumáticos está clara la superioridad neumática ${ }^{7,12,18,22}$, son hasta el momento los TQ más efectivos y más seguros en el mercado, las altas presiones generadas se pueden medir y permiten la compresión arterial controlada. La Food and Drug Administration considera a los TQ neumáticos quirúrgicos como clase I y los neumáticos de uso en combate clase II, de modo que plantean un mínimo riesgo para el paciente, permiten controlar la presión, menor daño tisular, brazaletes más amplios y ajuste primario rápido características que provocan menos dolor al paciente y no representan una fuente razonable de lesión durante el uso habitual; estas características deben incluirse en más TQ de campo civil y militar ${ }^{23,24}$. El trinquete se introdujo en tres prototipos desde 2005, todos presentaron fallas mecánicas y mal ajuste primario como ocurrió con el MAT, sin embargo este mecanismo de acción (safe-clip) es efectivo montado en un sistema de ajuste primario más eficiente como el $\mathrm{RMT}^{7,15,18}$. No hemos encontrado estudios que comparen los modelos THOR y STAT. Consideramos que STAT es el más sencillo de todos los $T Q$, ambos momentos (ajuste primario y mecanismo de acción) están presentes durante su ajuste en la extremidad, sin embargo debería ser más ancho y podría ser difícil detener el sangrado en la aplicación a una sola mano, motivado por la ausencia de una ventaja mecánica como la brinda el molinete o las palancas.

Desde 2011 se sabe que las hemorragias de la unión tienen vital importancia en los escenarios hostiles, destacando la hemorragia en la región pélvica como la más común, incluyendo nalgas e ingle proximal superior a la región del triángulo de escarpa. Gracias a los informes históricos y estudios recientes sabemos que Ios TQ troncales son dispositivos plausibles para controlar la hemorragia de unión en el campo de batalla ${ }^{25}$. Durante un estudio fisiológico en cerdos se registraron aumentos en la presión arterial y la frecuencia cardiaca con el uso del AAJT (dispositivo mixto neumático/molinete), también registraron paro respiratorio y complicaciones después de la retirada del dispositivo como hiperpotasemia y acidosis metabólica, factores que deben ser tomados en cuenta para el uso, diseño y futuro de estos dispositivos ${ }^{26,27}$. A pesar de que la última revisión de shock hemorrágico indica que en los sitios de la unión se aplique presión directa, uso de agentes y gasas hemostáticas, el uso de TQ de extremidades y de la unión debe continuar en el tratamiento del shock hemorrágico ${ }^{28}$, tanto en el campo de batalla como en el ámbito civil donde el tiempo apremia.

\section{Conclusiones}

Los TQ son fundamentales en el control del sangrado y han tenido cambios importantes en el diseño del sistema de ajuste primario gracias al mecanismo de palanca. El mecanismo de acción no ha tenido cambios sustanciales, reconocemos cinco tipos donde podemos agrupar todos los TQ hasta el momento diseñados, proponemos a la comunidad médica que se agrupen según su mecanismo de acción como sistemas de molinete, neumáticos, rolled-up, safe-clip y tornillo de compresión, según su sitio de aplicación como TQ de unión y $T Q$ de extremidades. Los mecanismos de acción más seguros y eficientes según la bibliografía para TQ de extremidades son: neumáticos, molinete, safe-clip y rolled-up. No identificamos estudios comparativos que demuestren superioridad entre $T Q$ de la unión. La clasificación puede ayudar a determinar cuál es el mejor de acuerdo con el contexto en que se encuentre el personal (militar, hospitalario, prehospitalario, escuelas, oficinas o espacios públicos). La comprensión del usuario sobre el funcionamiento de los dispositivos y su mecanismo de acción ayudará a obtener mejores resultados en la elección de este, 
facilitará su investigación, fabricación e innovación tecnológica, haciéndolos más seguros para evitar más muertes por shock hemorrágico.

\section{Financiamiento}

El siguiente estudio no ha recibido financiamiento alguno. El material adquirido para su análisis fue proporcionado por Carlos Antonio Contreras Cordero, quien lo adquirió por sus propios medios para la realización de este manuscrito.

\section{Conflicto de intereses}

Los autores declaran no tener ningún conflicto de intereses derivado del presente manuscrito.

\section{Bibliografía}

1. Thigh tourniquet, Roman, 199 BCE-500 CE [Internet]. Science Museum Group [consultado el 19/06/2018] Disponible en: https://collection.sciencemuseum.org.uk/objects/c086342/thigh-tourniquet-roman-199-bce-500ce-tourniquet

2. Kam PC, Kavanagh R, Yoong FF. The arterial tourniquet: Pathophysiological consequences and anaesthetic implications. Anaesthesia. 2001;56(6):534-45

3. Klenerman L. The tourniquet in surgery. J Bone Joint Surg Br.1962; 44-B:937-43.

4. Mabry RL, Holcomb JB, Baker AM, Cloonan CC, Uhorchak JM Perkins DE, et al. United States Army Rangers in Somalia: an analysis of combat casualties on an urban battlefield. J Trauma. 2000; 49(3): 515-28; discussion 28-9.

5. Bellamy RF. The causes of death in conventional land warfare: implications for combat casualty care research. Mil Med. 1984;149(2):55-62.

6. Brian J, East Ridge, Robert L. Death on the battlefield (2001-2011) Implications for the future of combat casualty care. J Trauma Acute Care Surg. 2012;73(6):431-7.

7. Walters TJ, Wenke JC, Greydanus DJ. Laboratory evaluation of battlefield tourniquets in human volunteers. U. S. Army Institute of Surgical Research,Texas, Fort Sam Houston; 2005.

8. Altamirano M, Kragh JJ, Aden J, Dubick M. Role of the windlass in improvised tourniquet use on a manikin hemorrhage model. J Spec Oper Med. 2015;2(15):42-6.
9. McHenry M. There is no trade-off between speed and force in a dynamic lever system. Biol Lett. 2011;7(3):386-6.

10. Cushing $\mathrm{H}$. Pneumatic tourniquets: with special reference to their use in craniotomies. Medical News. 1904;84:557.

11. Kragh Jr, Walters TJ, Baer DG, Fox CJ, Wade CE, Salinas J, et al. Survival with emergency tourniquet use to stop bleeding in major limb trauma. Ann Surg. 2009;249(1):1-7.

12. McEwen JA, Inkpen K. Surgical tourniquet technology adapted for military and prehospital use. MP-HFM. 2014;109:19.

13. Kragh JF Jr, Walters TJ, Baer DG, Fox CJ, Wade CE, Salinas J, et al. Practical use of emergency tourniquets to stop bleeding in major limb trauma. J Trauma. 2008;64(Suppl 2):S38-50.

14. Kragh JF, Kotwal RS, Cap AP. Performance of junctional tourniquets in normal human volunteers. Prehosp Emerg Care. 2015;19(3):391-8.

15. Wall PL, Sahr SM, Buising CM. Different width and tightening system emergency tourniquets on distal limb segments. J Spec Oper Med. 2015;15(4):28-38.

16. Stop the Bleed campaign [Internet]. American College of Surgeons; 2017. Disponible en: http://www.bleedingcontrol.org.

17. Kragh JF Jr, O'Neill ML, Walters TJ, Dubick MA, Baer DG, Wade CE et al. The military emergency tourniquet program's lessons learned with devices and designs. Mil Med. 2011;176(10):1144-52.

18. McKeague AL, Cox D. Phase I joint operational evaluation of field tourniquets [Internet]. Naval Medical Research Unit San Antonio, Combat Casualty Care Research Department; 2013. Disponible en: https://www. ratchetingbuckles.com/wp-content/uploads/2015/07/EVAL-2012-12Phase I-m2-.pdf

19. Roy Dory, D. Duane Cox, Bridget Endler, MS. Evauation of extremity tourniquets in the hands of non-medical personnel in simulated field conditions. Naval Medical Research Unit San Antonio; 2015.

20. Kragh JF Jr, Newton NJ, Tan AR. New and established models of limb tourniquet compared in simulated first aid. J Spec Oper Med. 2018; 18(2):36-41.

21. González-Alonso V, Orbañanos-Peiro L, Gómez-Crespo JM. Estudio del torniquete de dotación del Ejército de Tierra. Sanid Mil. 2016;72(2):87-94.

22. Taylor DM, Vater GM, Parker PJ. An evaluation of two tourniquet systems for the control of prehospital lower limb hemorrhage. J Trauma. 2011; 71(3):591-95.

23. Noordin S, McEwen JA, Kragh JF Jr. Reseña sobre conceptos actuales: Torniquetes quirúrgicos en Ortopedia. The Journal of Bone and Joint Surgery. 2009:1-13.

24. Saied A, Ayatollahi Mousavi A, Arabnejad F, Ahmadzadeh Heshmati A. Tourniquet in surgery of the limbs: A review of history, types and complications. Iran Red Crescent Med J. 2015;17(2):1-6.

25. Kragh JF Jr, Murphy C, Dubick MA, Baer DG, Johnson J, Blackbourne LH. New tourniquet device concepts for battlefield hemorrhage control. US Army Med Dep J. 2011 Apr-Jun:38-48.

26. Kragh JF, Kotwal RS, Cap AP. Performance of junctional tourniquets in normal human volunteers. Prehosp Emerg Care. 2015;19(3):391-8.

27. Kheirabadi BS, Terrazas IB, Miranda N. Physiological consequences of abdominal aortic and junctional tourniquet (AAJT) application to control hemorrhage in a swine model. Shock. 2016;46(3):160-6.

28. Longo DL. Hemorrhagic shock. N Engl J Med. 2018:370-79. 